\title{
ORIGINAL ARTICLE \\ In search of genetic constraints limiting the evolution of egg size: direct and correlated responses to artificial selection on a prenatal maternal effector
}

\author{
JL Pick, P Hutter and B Tschirren
}

Maternal effects are an important force in nature, but the evolutionary dynamics of the traits that cause them are not well understood. Egg size is known to be a key mediator of prenatal maternal effects with an established genetic basis. In contrast to theoretical expectations for fitness-related traits, there is a large amount of additive genetic variation in egg size observed in natural populations. One possible mechanism for the maintenance of this variation is through genetic constraints caused by a shared genetic basis among traits. Here we created replicated, divergent selection lines for maternal egg investment in Japanese quail (Coturnix japonica) to quantify the role of genetic constraints in the evolution of egg size. We found that egg size responds rapidly to selection, accompanied by a strong response in all egg components. Initially, we observed a correlated response in body size, but this response declined over time, showing that egg size and body size can evolve independently. Furthermore, no correlated response in fecundity (measured as the proportion of days on which a female laid an egg) was observed. However, the response to selection was asymmetrical, with egg size plateauing after one generation of selection in the high but not the low investment lines. We attribute this pattern to the presence of genetic asymmetries, caused by directional dominance or unequal allele frequencies. Such asymmetries may contribute to the evolutionary stasis in egg size observed in natural populations, despite a positive association between egg size and fitness.

Heredity (2016) 116, 542-549; doi:10.1038/hdy.2016.16; published online 9 March 2016

\section{INTRODUCTION}

The environment experienced during development can have a profound effect on survival and reproduction (Henry and Ulijaszek, 1996; Lindström, 1999). Mothers are often in a unique position to influence this early environment, and so can alter the developmental trajectory of their offspring, through a process known as maternal effects (Mousseau and Fox, 1998). Maternal effects arise from the phenotype of the mother acting on the environment of the offspring, and thereby its phenotype, and can therefore be influenced by both the mother's genes and the environment she experiences (Wolf et al., 1998). The former is of particular interest from an evolutionary perspective because it allows the traits causing maternal effects (hereafter referred to as maternal effectors) to evolve (Wolf et al., 1998). This in turn can greatly accelerate (positive maternal effects) or impede (negative maternal effects) the response to selection of the affected offspring trait (Kirkpatrick and Lande, 1989; Wolf et al., 1998).

In oviparous species, a large amount of attention has been focused on the egg size and its role as a maternal effector (Bernardo, 1996). In many taxa, egg size is strongly positively associated with offspring growth and survival in early life (McGinley et al., 1987; Fox and Czesak, 2000; Krist, 2011). Furthermore, many studies have shown egg size to be moderately to highly heritable (Christians, 2002; Fox and Czesak, 2000). However, despite being both heritable and associated with fitness, there is little evidence that egg size responds to contemporary selection in wild populations (for example, Hõrak et al., 1997). This apparent evolutionary stasis, along with high amounts of additive genetic variance, has been observed in many fitness-related traits (Houle, 1992; Merilä et al., 2001). One proposed solution to this paradox is that evolution is constrained by underlying genetic correlations, caused by a shared genetic basis among traits (hereafter referred to as genetic constraints; Merilä et al., 2001; Walsh and Blows, 2009). Consequently, in order to understand the capacity for this maternal effector to evolve in wild populations, it is necessary to understand its genetic relationships with other fitness-related traits.

Firstly, it is often assumed that larger eggs represent a larger supply of resource for developing offspring (Bernardo, 1996). However, egg size by itself is not necessarily a measure of maternal resource investment; larger eggs could, for example, contain more water rather than more lipids or proteins (Fischer et al., 2006). It is therefore unclear whether selection on the basis of egg size would result in a correlated response in maternal resource investment. Because the positive association between egg size and fitness is likely caused by these resources (for example, Finkler et al., 1998), egg size would only be expected to respond to selection in the wild if there are positive genetic correlations between the egg size and the maternal resource investment.

Secondly, life history theory predicts that an increase in per offspring investment should come at the cost of fecundity (Smith and Fretwell, 1974). The presence of this trade-off may help explain

Department of Evolutionary Biology and Environmental Studies, University of Zurich, Zurich, Switzerland

Correspondence: Dr J Pick, Department of Evolutionary Biology and Environmental Studies, University of Zurich, Winterthurerstrasse 190, Zurich 8057, Switzerland.

E-mail: joel.I.pick@gmail.com

Received 10 November 2015; revised 29 January 2016; accepted 29 January 2016; published online 9 March 2016 
the maintenance of variation in egg size in wild populations. Such a trade-off may occur as a consequence of resource limitation or via an underlying genetic constraint. Although many studies have focused on energetic trade-offs, the evidence for a negative genetic correlation between offspring number and per offspring investment is equivocal in many taxa (Lessells et al., 1989; Bernardo, 1996; Schwarzkopf et al., 1999; Czesak and Fox, 2003; Fischer et al., 2006).

Finally, egg size might simply be a function of body size (Fox and Czesak, 2000). As there is evidence that there are substantial forces constraining the evolution of larger body size (Blanckenhorn, 2000), if a strong genetic correlation between egg size and body size exists, the evolution of egg size would be constrained by the same forces as the evolution of body size. Consequently it is important to estimate the strength of the genetic correlation between the egg size and the body size to determine if the egg size can evolve independently (Czesak and Fox, 2003).

A powerful way to experimentally test the potential for evolutionary change, as well as for possible genetic constraints, is through artificial selection (Conner, 2003). Artificial selection lines for egg size have been previously established in invertebrates (Schwarzkopf et al., 1999; Czesak and Fox, 2003; Fischer et al., 2006), but the approach is still rarely used in vertebrates because of their often long generation times. Here we created replicated, divergent selection lines for relative egg size (that is, egg size corrected for female body size) in a captive population of a precocial bird, the Japanese quail (Coturnix japonica). On the basis of these lines, we demonstrate that selection on egg size results in a correlated response in maternal resource investment, and that the evolution of egg size is not constrained by genetic correlations with either fecundity or body size. The response to selection, however, was asymmetrical, which is potentially the result of genetic asymmetries. We suggest that such genetic asymmetries may play an important role in mediating dynamics of egg size evolution in the wild.

\section{METHODS}

\section{Study population and selection lines}

This study was conducted using a captive population of Japanese quail maintained at the University of Zurich, Switzerland. Males and females were housed in separate outdoor aviaries; females in a single sex aviary, and males in a mixed sex aviary together with non-experimental females (both $7 \times 5.5 \mathrm{~m}$ ). The founder population (generation 0), consisting of 189 birds ( 91 females and 98 males), was obtained from a commercial quail egg farm located in the southeast of Switzerland, where birds from two different origins were maintained in two separate populations. These populations had been maintained since 1998 at the farm before our experiment began in 2012, and no (intentional) artificial selection had been imposed on the birds during this time. Although no pedigree was available for the founders, large populations were maintained on the farm, and efforts are made to avoid inbreeding, meaning that the starting stock had a large effective population size. To further increase genetic diversity in our study population, we initially crossed birds from the two origins and used these crosses as the starting population for our selection experiment (generation 1). These birds were randomly split into two replicates consisting of 34 and 38 male-female pairs, respectively (see below for details about the pairings).

We then created replicated, divergent selection lines for high and low maternal egg investment, using relative egg size as the selection criterion. We used relative egg size rather than absolute egg size as the selection criterion in order to not simply select based on body size, but rather based on the investment a female makes in her eggs independent of her size. Relative egg size was determined as the residuals from a regression of egg size (measured in grams egg mass) against female body size (that is, the first principle component of female body mass and tarsus length; PC1 explained 0.571-0.917 \% of variation across generations). These residuals were recalculated for each line, replicate and generation. Relative egg size was highly correlated with absolute egg size in all lines, replicates and generations (mean $r \pm$ s.d., $0.884 \pm 0.120$, $n=14)$, but, as expected, not with female tarsus length $(-0.002 \pm 0.143, n=14)$ or body mass $(0.002 \pm 0.143, n=14)$. The correlation between absolute and relative egg size did not change over the course of the experiment (see Supplementary Material S1).

In generation 1, the 10 females with the largest and smallest relative egg sizes were assigned to the high and low investment line, respectively, in both replicates. In each of the subsequent three generations (that is, generations 2-4), we selected the most extreme 10 pairs $(50 \%)$ in both the high and low lines of both replicates. The eggs of the selected pairs were collected, incubated and hatched as outlined below. Two sons and two daughters from each selected pair were then used for the next breeding round, giving 20 breeding pairs per line, replicate and generation. If the selected pairs did not have enough sons and daughters, we used offspring from the 11th and 12th ranked pairs to make up the total of 20 pairs within a line replicate. Within a replicate, the high and low investment lines were always bred simultaneously to control for seasonal and age effects. In addition, an unselected base population was maintained in the same facility, originating from the same founder populations. These birds were not bred at the same times or ages as the selection lines and so are not directly comparable. Nevertheless, given that there was no directional change in mean egg size over five generations in this unselected population $\left(F_{1,3}=0.51\right.$, $P=0.528$; see Supplementary Figure S2), we can exclude the possibility that systematic changes in egg size have occurred over time due to inadvertent effects of husbandry.

\section{Breeding protocol}

For breeding, males and females were brought into cages $(122 \times 50 \times 50 \mathrm{~cm})$ in our breeding facility for 3-4 weeks, and body mass and tarsus length were measured (to the nearest $1 \mathrm{~g}$ and $0.1 \mathrm{~mm}$, respectively). Our facility is kept on a 16:8 light:dark cycle at $\sim 20^{\circ} \mathrm{C}$. Cages contained ad libitum food, water, grit, a source of calcium, a house and a raised sand bath. The bottom of the cages was filled with sawdust. We kept one male-female pair per cage. Breeding pairs consisted of non-related individuals from the same line and replicate. Nonrelated was defined as individuals not sharing any grandparents. This resulted in no quail having an inbreeding coefficient $>0.016$ by generation 4 (assuming that the initial population consisted of unrelated individuals). All individuals were bred once, with the exception of birds from generation 4, which were let back into the aviary for 7-9 weeks after the first breeding round and then brought back into breeding cages, where they were bred with different partners.

Eggs were collected every morning over a period of 14 days. They were labeled with a non-toxic marker and weighed (to the nearest $0.01 \mathrm{~g}$ ). The first two eggs were dissected (see below) and the middle eggs (from the sixth and seventh days) were frozen. The remaining eggs were stored at $12{ }^{\circ} \mathrm{C}$ before being artificially incubated (Favorit, HEKA Brutgeräte, Rietberg, Germany). During the first 14 days, eggs were incubated at $37.8^{\circ} \mathrm{C}$ and $55 \%$ humidity. They were then candled and all developed eggs were transferred to a hatcher (Favorit, HEKA Brutgeräte) in individual compartments, and kept at $37.6{ }^{\circ} \mathrm{C}$ and $80 \%$ humidity until hatching. Hatchlings were marked with an individually numbered plastic leg ring and kept in a heated cage $(109 \times 57 \times 25 \mathrm{~cm}$, Kükenaufzuchtbox Nr 4002/C, HEKA Brutgeräte) for 2 weeks. For the first 5 days, the temperature was kept at $35-38{ }^{\circ} \mathrm{C}$, then slowly lowered to $25^{\circ} \mathrm{C}$ over the next 9 days. After 2 weeks, the chicks were transferred to cages within our breeding facility. At 4 weeks of age, they were sexed according to their plumage and put into the outdoor aviaries.

\section{Laying rate and laying intervals}

As quails are indeterminate layers (Cole, 1917), removing an egg every day (as necessary to successfully store and artificially incubate eggs) causes the females to continually lay eggs. Therefore, we cannot directly measure clutch size in our system, and we used an indirect measure of fecundity instead: the proportion of days on which a female laid an egg while in her cage (hereafter referred to as laying rate), a measure that is commonly used to quantify fecundity in poultry (for example, Wright et al., 2012). Females may be constrained in how fast egg nutrients can be deposited, resulting in larger eggs taking longer to produce (for example, Meijer, 1992). Under this scenario, we would predict an increase in the time between two eggs, as well as in the number of laying gaps (Williams, 
2012, and references therein) in the high investment line. In our population, most females lay between 6 and 10 eggs in a 10-day period (JLP, personal observation). This range in laying rate is equivalent to the variation in clutch size seen in many natural populations (Johnsgard, 1988). For females in generation 4, we furthermore recorded the time between laying two eggs (hereafter referred to as laying interval); during the third week, the females were in cages. All cages were checked every hour up until 1 hour before lights off (21:00), recording the hour in which an egg was found in a cage. This was done 3 or 4 days in a row to ensure that two eggs were collected from each female on consecutive days. Eggs found in the morning were assumed to have been laid in the hour before lights off.

In generation 5, we brought fewer females into cages than in previous generations and for a shorter period of time (some for only 1 week). We therefore did not quantify laying rate in this generation, and the sample size in generation 5 was reduced compared with previous generations.

\section{Egg measurements}

Starting from the third generation, one or two eggs were dissected from each female. Wet yolks were weighed and shells were dried at $80{ }^{\circ} \mathrm{C}$ until a constant weight and weighed (both to nearest $0.001 \mathrm{~g}$ ). Albumen mass was calculated as the total egg mass minus yolk and shell masses. For generation 4, we furthermore separated eggs into yolk, albumen and shell, weighed (wet mass) and dried them in a drying oven at $80^{\circ} \mathrm{C}$ for a minimum of $15 \mathrm{~h}$ and weighed them again (dry mass). Dry masses of eggs laid on the first 2 days, as well as on the fifteenth and sixteenth days the females were in cages were determined in this generation, to test the consistency of egg composition across the laying sequence. Egg size and all egg components were highly repeatable within females (Supplementary Table S1). Differences between eggs laid at the beginning and the end of a laying sequence are described in the Supplementary Material S3.

All procedures were conducted under licenses provided by the Veterinary Office of the Canton of Zurich, Switzerland (permit numbers 195/2010; 14/2014; 156).

\section{Statistical analyses}

Response to selection and realized heritability. For the analysis of the response to selection, we followed the methods outlined by Falconer and Mackay (1996). Using the breeder's equation

$$
R=h^{2} S
$$

where $R$ is the response to selection, $S$ is the selection differential and $h^{2}$ is the narrow-sense heritability, we calculated the realized heritability $\left(h_{\mathrm{r}}^{2}\right)$, defined as the heritability as realized from the response to selection (Falconer and Mackay, 1996). Our experiment ran over multiple generations, which allowed us to estimate $h_{\mathrm{r}}^{2}$ and its associated error by regressing the cumulative response to selection $\left(R_{\mathrm{C}}\right)$ against the cumulative selection differential $\left(S_{\mathrm{C}}\right)$ and forcing the intercept through 0 (the difference between the two lines in the initial population), giving

$$
R_{\mathrm{C}}=b S_{\mathrm{C}}
$$

where $b=\frac{1}{2} h_{\mathrm{r}}^{2}$, as selection was only on female phenotypes and we assume autosomal inheritance (Falconer and Mackay, 1996). The cumulative response to selection is a robust way to assess selection response, as it is a means to overcome variation between generations because of, for example, environmental fluctuations that can distort the interpretation of per-generation responses to selection (see Falconer and Mackay, 1996, pp 194-198). $R$ was calculated as the mean egg size of the offspring generation minus the mean egg size of the parental generation (egg size is defined here as absolute egg size; relative egg size was calculated within generations and thus is inappropriate for between-generation comparisons). $S$ was calculated as the mean egg size of selected mothers minus the mean egg size of the entire parental generation. We corrected for reproductive difference between females (that is, having different numbers of offspring) by weighting the mean of selected parents by the number of daughters per female in the next generation (effective selection differential; Falconer and Mackay, 1996). Egg size was standardized (mean $=0$, s.d. $=1$, across all data) before calculation of $R$ and $S$, meaning that $S$ is equivalent to the

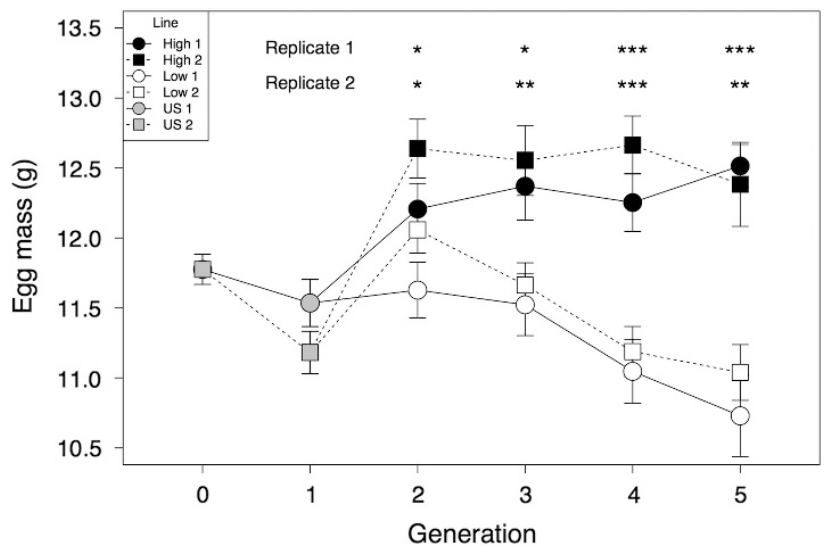

Figure 1 Absolute egg size in the selection lines over the course of six generations (Mean \pm s.e.m.). US represents the unselected founder population. Stars represent the difference between high and low maternal investment lines in each replicate and generation $\left({ }^{*} P<0.05,{ }^{*} P<0.01\right.$, $* * * P<0.001)$.

selection intensity on females $\left(i_{f}\right)$ and the selection intensity $(i)$ is equal to $\frac{1}{2} i_{f}$ (Falconer and Mackay, 1996).

As we bred the quail throughout the year, there were substantial environmental differences between generations and replicates in some measured traits (see Figure 1 and Supplementary Figure S2). Given that we bred both lines within a replicate at the same time, we can control for these seasonal effects by using the two lines within a replicate as controls for each other (Hill, 1972; Falconer and Mackay, 1996). Therefore, $R_{\mathrm{d}}$ and $S_{\mathrm{d}}$ were calculated as the differences in $R$ and $S$ between the lines within each generation and replicate. $R_{\mathrm{d}}$ and $S_{\mathrm{d}}$ were then used to calculate $R_{\mathrm{C}}$ and $S_{\mathrm{C}} . R_{\mathrm{C}}$ and $S_{\mathrm{C}}$ therefore represent cumulative differences between the lines. We tested for a difference in $h_{\mathrm{r}}^{2}$ between the two replicates, through testing for an interaction between $S_{\mathrm{C}}$ and replicate on $R_{\mathrm{C}}$. We also calculated an overall $h_{\mathrm{r}}^{2}$ by pooling the two replicates.

As $h_{r}^{2}$ was calculated using the differences between the two lines, we could not test directly for differences in $h_{r}^{2}$ between the lines. As we were interested in testing for the presence of an asymmetric response to selection, we also tested for an interaction between line and $S_{\mathrm{C}}$ on $R_{\mathrm{C}}$, where $R_{\mathrm{C}}$ and $S_{\mathrm{C}}$ were calculated from $R$ and $S$, including data from both lines and replicates. Replicate was included as a factor in the model. This analysis was performed for generations $2-5$, as the mean egg size in generation 1 was clearly different from other generations. As it has been suggested that heritability may not represent the most informative metric of evolutionary potential (Houle, 1992), we also calculated the coefficient of additive genetic variance $\left(C V_{A}\right)$ as

$$
C V_{\mathrm{A}}=\frac{\sqrt{h^{2} V_{\mathrm{P}}}}{\mu}
$$

where $\mu$ is the phenotypic mean and $V_{\mathrm{P}}$ is the phenotypic variance, and evolvability $\left(I_{\mathrm{A}}\right)$ as $C V_{\mathrm{A}}^{2}$.

Correlated responses to selection and realized co-heritabilities. For wet yolk mass, wet albumen mass, dry shell mass, yolk/albumen ratio, laying rate, female body mass and female tarsus length, we had information over enough generations to calculate the co-heritability of these traits with egg size. Co-heritability $\left(r_{\mathrm{A}} h_{X} h_{Y}\right)$ is the strength to which one trait $(Y)$ responds to selection on another trait $(X)$ (that is, the correlated response) and is a composite of the heritability of the two traits and the genetic correlation between them (Falconer and Mackay, 1996). Co-heritability can be calculated from

$$
C R_{Y}=r_{\mathrm{A}} h_{X} h_{Y} S_{X} \frac{\sigma_{P Y}}{\sigma_{P X}}
$$

where $r_{\mathrm{A}}$ is the genetic correlation between trait $X$ and $Y, h$ is the square root of the heritability of a trait, $C R_{Y}$ is the correlated response of trait $Y$ to selection on trait $X$ and $\sigma_{P}$ is the s.d. of a trait (derived from Falconer and Mackay, 1996, equation (19.6)). 
As with the realized heritability, given that we had data over multiple generations, we estimated co-heritability between a trait and egg size from the regression of cumulative correlated response in that trait $\left(C R_{C Y}\right)$ against the cumulative selection differential of egg size $\left(S_{C X}\right)$. To calculate $C R_{Y}$ and $S_{X}$, we again used the difference between high and low lines within each generation and replicate. For these calculations, we standardized all variables (mean $=0$, s.d. $=1$, across all data), meaning that $\frac{\sigma_{P Y}}{\sigma_{P X}}=1$. Therefore, the slope of the regression is equal to the co-heritability and as selection was only on female phenotypes this estimate (and s.e.m.) was doubled (Falconer and Mackay, 1996). We tested for a difference in co-heritability between the two replicates, through an interaction between $S_{C X}$ and replicate. We also calculated an overall co-heritability by pooling the data from the two replicates. Furthermore, we tested for a difference in co-heritability among egg traits by testing for an interaction between $S_{C X}$ and trait using pooled data from both replicates. Significance of pairwise differences between these traits was obtained by calculating a $t$ statistic from the estimated difference between slopes. After visual inspection of the data, we also tested for a nonlinear co-heritability for body mass and tarsus length, by including a quadratic $S_{C X}$ term and pooling replicates.

Dry egg components and laying behavior. In generation 4, we additionally tested for differences between the lines in the mass of dry egg components and in laying intervals. We ran linear models including line and replicate as fixed factors. For the dried egg components data, the mean values for each constituent (yolk, albumen, shell and total dry mass) were calculated from all measured eggs of a female. We also ran models to correct for possible allometric relationships of the dry egg constituents with egg size and body size. The response variables were log transformed, and included log transformed body size (tarsus length cubed) or egg size as a covariate. Given that we have selected on relative egg size, we would expect a larger amount of dry components in the high investment line when controlling for body size, but no difference between the lines when controlling for egg size.

For the laying interval data, we calculated a mean value for all females for which we had more than one laying interval. Given that we could not be completely sure that eggs laid after the cages were last checked were laid in the hour before dark, we also ran an analysis excluding any laying intervals that were calculated including that hour. One high-line female was excluded from both analyses as her laying interval was $>4$ s.d. out of the range shown by other females.

In addition to the analyses described above, we also present the results of $t$-tests of difference in all traits between the lines in generation 4 (replicates pooled). All analyses were run in R (3.0.3, R Core Team, 2014).

\section{RESULTS}

\section{Response to selection}

The selection intensity $(i)$ was $0.344 \pm 0.105$ (mean \pm s.d.) in the high investment line and $-0.201 \pm 0.087$ in the low investment line. After only one generation of selection, the lines differed significantly in absolute egg size (Figure 1 and Supplementary Table S3). This response increased as the selection experiment progressed as demonstrated by the high $h_{\mathrm{r}}^{2}$ (Table 2 and Figure $2 \mathrm{a}$ ). By generation 4, there was a 1.25 s.d. difference in absolute egg size between the lines (Table 1 and Figure 1). Although the response to selection was strong in both replicates, it was weaker in the second replicate, with a significantly lower $h_{\mathrm{r}}^{2}$ (Table 2 and Figure 2a). Using $h_{\mathrm{r}}^{2}$ we estimated $C V_{\mathrm{A}}$ as 0.075 (replicate 1: 0.081 ; replicate 2: 0.071 ) and $I_{\mathrm{A}}$ as 0.0056 (replicate 1: 0.0066; replicate 2: 0.0050 ). When comparing the response to selection between the two lines, we found a significant difference $\left(S_{C} \mathrm{x}\right.$ line: $\left.F_{1,11}=13.37, P=0.004\right)$, with a strong response in the low investment line $\left(F_{1,5}=41.63, P=0.001\right)$, but no apparent response in the high investment line $\left(F_{1,5}=0.12, P=0.746\right)$, after the first generation of selection.

\section{Correlated responses to selection in egg components}

All egg components showed a strong and significant correlated response to selection on egg size (Table 2 and Figure 2). The differences between lines in generation 4 are shown in Table 1 . Overall egg components differed in the strength of their correlated response to selection on egg size $\left(S_{C X} \mathrm{x}\right.$ trait: $F_{2,21}=7.85, P=0.003$, Figure 2). The co-heritability of albumen mass was significantly higher than that of both yolk mass $\left(t_{21}=2.28, P=0.033\right)$ and shell mass $\left(t_{21}=3.95, P<0.001\right)$, but there was no significant difference between yolk mass and shell mass $\left(t_{21}=1.66, P=0.111\right)$. The correlated response of shell mass did not differ between replicates. However yolk mass responded significantly less in the second replicate and there was a tendency for the same pattern in albumen mass (Table 2). Yolk/albumen ratio did not show a correlated response to selection on egg size (Table 2).

To test if these correlated responses of egg components correspond to changes in maternal resource investment, we examined the dry egg constituents. As expected, high-line eggs had absolutely more dry constituents than low-line eggs (Tables 1 and 3). All dry components had a significant positive relationship with egg size and all but dry yolk mass had a significant positive relationship with body size (Table 3 ). Eggs from the high investment lines contained more dry components than eggs from the low investment lines when correcting for body size. When correcting for egg size, however, eggs from the high investment lines contained less yolk and more albumen (Table 3) and the two lines did not differ in shell or total dry mass (Table 3). Both dry albumen and yolk masses correlated strongly with wet albumen and yolk masses respectively (albumen: $r=0.938, P<0.001, N=80$; yolk: $r=0.996, P<0.001, N=80)$.

\section{Correlated response to selection in laying behavior}

Laying rate showed no correlated response to selection on egg size, as shown by a non-significant co-heritability (Table 2). Similarly there was no difference between the lines in laying interval $\left(F_{1,76}=0.770, P=0.383\right.$, Table 1$)$ and no difference between the replicates $\left(F_{1,76}=0.376, P=0.542\right)$. When excluding laying intervals where one of the laying times was inferred (see Methods) the results were qualitatively similar (line: $F_{1,60}=0.424, P=0.518$; replicate: $\left.F_{1,60}=1.015, P=0.318\right)$.

\section{Correlated response to selection in body size}

Both tarsus length and body mass showed a significant nonlinear correlated response to selection on egg size, with an initial strong correlated response that declined during the course of the selection experiment (Table 2 and Figures $2 \mathrm{c}$ and $\mathrm{d}$ ).

\section{DISCUSSION}

We show that maternal egg investment responds rapidly to directional selection, with lines differing in egg size by more than 1 s.d. after only four generations of selection. This rapid response of egg size to selection is remarkable given the relatively weak selective pressure that was applied to female phenotypes only, with the mean selection intensity being well within the range seen in natural populations (Kingsolver et al., 2001). Also, selection only on females reflects what would occur in the wild, where selection acts only upon individuals expressing a phenotype. If variation in egg size is associated with variation in fitness, as is commonly found (Krist, 2011), egg size should respond rapidly to selection in wild population.

Although intuitive, it is not inevitable that selection on egg size results in a correlated response in resource investment (Fischer et al., 2006). In our selection experiment, a strong correlated response in 

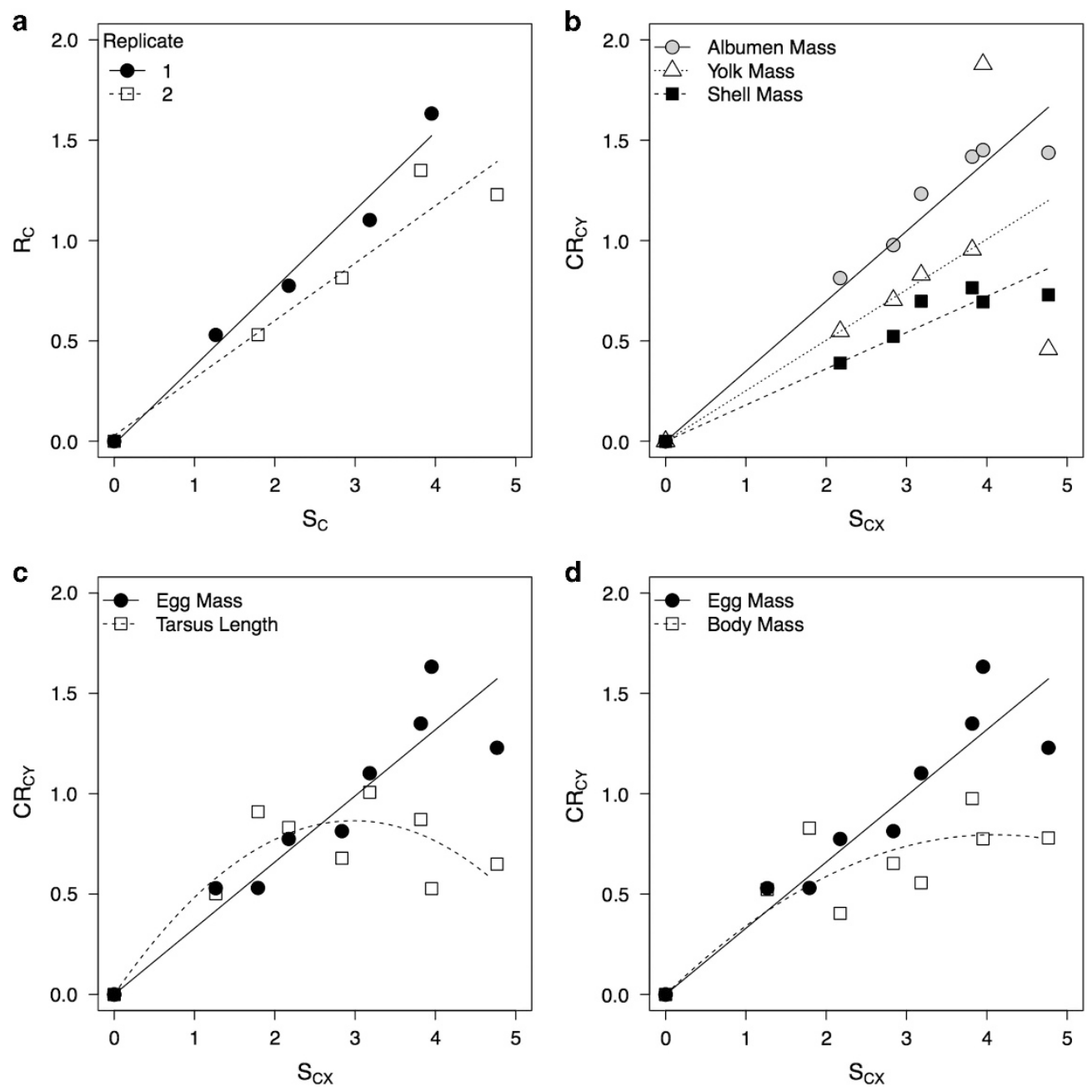

Figure 2 Response to selection of (a) absolute egg size in the two replicates, (b) yolk, albumen and shell mass (replicates pooled), (c) absolute egg size and tarsus length and (d) absolute egg size and body mass. Both axes are in units of s.d. $S_{C X}$ is the cumulative selection differential on absolute egg size between the lines, $R_{\mathrm{C}}$ is the cumulative response to selection in absolute egg size between the lines and $\mathrm{CR}_{C Y}$ is the cumulative correlated response to selection between the lines in traits indicated by the legends. Lines represent estimates of realized (co-)heritability.

yolk, albumen and shell mass was observed. Furthermore, eggs from the high investment lines contained more dry components, which are almost entirely lipids and proteins in yolk, proteins in albumen and calcium in the shell (Romanoff and Romanoff, 1949). All of these resources are vital to the successful development of the chick (Finkler et al., 1998). Producing larger eggs thus constitutes a higher resource investment by the mother.

Eggs from the high maternal investment lines had more dry albumen than eggs from the low maternal investment line, even after controlling for egg size. Furthermore, albumen mass responded more strongly to selection on egg size than did yolk mass. This is in line with previous findings in chickens, where selection on yolk/albumen ratio resulted in a larger response in albumen mass than yolk mass (Miyoshi et al., 1996). Albumen consists of a high proportion ( $50 \%)$ of egg protein in precocial species (Carey et al., 1980) and because albumen is the main protein source for the chick during prenatal development (Freeman and Vince, 1974) it is likely a limited factor during critical periods early in life.

Although we here show that egg size can rapidly respond to selection and results in a correlated response in resource investment, evolutionary stasis in egg size is observed in many natural populations.
One mechanism that could contribute to this phenomenon is a size/ number trade-off, which is a key concept in life history theory (Smith and Fretwell, 1974). To date, however, there is surprisingly little empirical evidence for such a constraint at the genetic level (Bernardo, 1996; Schwarzkopf et al., 1999; Czesak and Fox, 2003; Fischer et al., 2006). In wild bird populations, for example, so far only three studies have tested for a genetic correlation between egg size and clutch size (Lessells et al., 1989; Garant et al., 2008; Santure et al., 2013), two of which were performed in the same population (Garant et al., 2008; Santure et al., 2013). The results of these studies were equivocal with only one showing a significant negative genetic correlation (Garant et al., 2008). Similarily, no correlated response in egg size was observed in Japanese quail artificially selected for lay rate (Nestor et al., 1983). In line with these previous findings, we found no differences in laying rate or laying intervals between the two selection lines, suggesting that females of the high and low investment lines produced differently sized eggs in the same amount of time (see also Christians and Williams, 2001). Although we could only use an indirect measure of fecundity in our study, this result suggests that there is not an inevitable genetic trade-off between egg size and egg number. Additional studies in wild bird populations that test for a (lack of a) 
genetic correlation between egg size and clutch size are, however, required to confirm that the two life history traits can evolve independently under natural conditions.

Given that we fed our birds ad libitum, we cannot rule out the presence of an energetic trade-off when resources are limited (McGinley et al., 1987; Czesak and Fox, 2003), as is the case in most wild populations. However, the presence of an energetic trade-off is also largely unsupported in wild-living birds. For example, manipulations forcing birds to lay additional eggs do not necessarily result in smaller replacement eggs (Nager et al., 2000; Williams and Miller,

Table 1 Average phenotypes and testing for differences between selection lines for high and low maternal egg investment

\begin{tabular}{|c|c|c|c|c|c|}
\hline Trait & High & Low & $t$ & $d f$ & $\mathrm{P}$ \\
\hline \multicolumn{6}{|l|}{ Egg size } \\
\hline Egg size (g) & $12.46 \pm 0.94$ & $11.12 \pm 0.91$ & 6.49 & 78 & $<0.001$ \\
\hline Dry mass (g) & $3.857 \pm 0.334$ & $3.411 \pm 0.325$ & 6.04 & 78 & $<0.001$ \\
\hline \multicolumn{6}{|l|}{ Egg components } \\
\hline Wet albumen (g) & $7.851 \pm 0.570$ & $6.879 \pm 0.523$ & 7.95 & 78 & $<0.001$ \\
\hline Dry albumen (g) & $0.912 \pm 0.088$ & $0.789 \pm 0.070$ & 6.96 & 78 & $<0.001$ \\
\hline Wet yolk (g) & $3.620 \pm 0.413$ & $3.228 \pm 0.365$ & 4.49 & 78 & $<0.001$ \\
\hline Dry yolk (g) & $1.899 \pm 0.229$ & $1.699 \pm 0.213$ & 4.05 & 78 & $<0.001$ \\
\hline Dry shell (g) & $1.044 \pm 0.090$ & $0.922 \pm 0.077$ & 6.51 & 78 & $<0.001$ \\
\hline Yolk/albumen ratio & $0.461 \pm 0.042$ & $0.469 \pm 0.036$ & 0.87 & 78 & 0.387 \\
\hline \multicolumn{6}{|l|}{ Fecundity } \\
\hline Laying rate & $0.915 \pm 0.034$ & $0.922 \pm 0.044$ & 0.71 & 78 & 0.478 \\
\hline Laying interval (hours) & $24.18 \pm 0.55$ & $24.07 \pm 0.55$ & 0.88 & 77 & 0.383 \\
\hline \multicolumn{6}{|l|}{ Body Size } \\
\hline Tarsus length (mm) & $40.6 \pm 1.2$ & $39.3 \pm 1.4$ & 4.55 & 78 & $<0.001$ \\
\hline Body mass (g) & $281 \pm 24$ & $260 \pm 28$ & 3.57 & 78 & $<0.001$ \\
\hline
\end{tabular}

Mean \pm s.d. of traits measured in generation 4 are presented. Data from the two replicates were pooled. Significant differences are displayed in bold.
2003), and in one study the number of additional eggs laid was even positively correlated with egg size (Williams and Miller, 2003). Hormonal manipulations targeting the potential mechanistic basis of an egg size/number trade-off have also found equivocal results. For example, increasing plasma follicle stimulating hormone, which is known to mediate the egg number/size trade-off in lizards (Sinervo and Licht, 1991), resulted in a decrease in both egg size and clutch size (Christians and Williams, 2002).

Our measure of relative egg size was highly phenotypically correlated with absolute egg size, but not with body size. Therefore, at least on a phenotypic level, we were not directly selecting on body size. Nevertheless, we saw a strong initial response in body size, indicating that there was a strong underlying genetic correlation. This initial response mirrors what was seen in a selection experiment in chickens, where both selection on body size and on egg size resulted in a similar magnitude of response in egg size (Festing and Nordskog, 1967). Moreover, phenotypic correlations between egg size and body size were found to be much weaker than genetic correlations (Festing and Nordskog, 1967). This suggests that weak phenotypic correlations in the wild may hide potentially strong genetic correlations, which seems to be the case in our population.

Despite the strength of this initial correlated response in body size, it diminished rapidly as we selected further for relative egg size. This indicates that the genetic correlation between egg size and body size is either not stable, and/or that there is substantial additive genetic variation for relative egg size (Czesak and Fox, 2003). Furthermore, the increases in resource investment were not simply a function of larger body size. There was a strong difference between the lines in dry egg components, even after correcting for body size, and dry yolk mass was in fact not related to body size. Our results therefore show that it is indeed possible to select on relative resource investment, in contrast to the long held beliefs (Hutt, 1949).

Overall, we found little support for a role of body size or fecundity in constraining the evolution of egg size. We cannot exclude the possibility, however, that other genetic constraints or energetic tradeoffs, not assessed in our study, may constrain the evolution of

Table 2 Realized heritability of absolute egg size and co-heritabilities of different traits with absolute egg size (estimate \pm s.e.m.)

\begin{tabular}{|c|c|c|c|c|c|c|c|c|c|}
\hline & \multicolumn{4}{|c|}{ Pooled } & \multirow[t]{2}{*}{ Replicate 1} & \multirow[t]{2}{*}{ Replicate 2} & \multicolumn{3}{|c|}{ Difference } \\
\hline & Estimate & $F$ & $d f$ & $P$ & & & $F$ & $d f$ & $P$ \\
\hline \multicolumn{10}{|l|}{ Realized heritability } \\
\hline Absolute egg size & $0.660 \pm 0.038$ & 290.83 & 1,9 & $<0.001$ & $0.768 \pm 0.042$ & $0.588 \pm 0.034$ & 10.92 & 1,8 & 0.011 \\
\hline \multicolumn{10}{|l|}{ Egg components } \\
\hline Wet albumen mass & $0.698 \pm 0.024$ & 793.73 & 1,7 & $<0.001$ & $0.750 \pm 0.032$ & $0.664 \pm 0.026$ & 4.30 & 1,6 & 0.084 \\
\hline Wet yolk mass & $0.504 \pm 0.100$ & 25.23 & 1,7 & 0.002 & $0.738 \pm 0.118$ & $0.346 \pm 0.096$ & 6.77 & 1,6 & 0.041 \\
\hline Dry shell mass & $0.362 \pm 0.018$ & 448.65 & 1,7 & $<0.001$ & $0.382 \pm 0.028$ & $0.348 \pm 0.022$ & 0.93 & 1,6 & 0.371 \\
\hline Yolk/albumen ratio & $-0.072 \pm 0.108$ & 0.45 & 1,7 & 0.526 & $0.162 \pm 0.136$ & $-0.230 \pm 0.112$ & 4.91 & 1,6 & 0.069 \\
\hline \multicolumn{10}{|l|}{ Fecundity } \\
\hline Laying rate & $-0.056 \pm 0.038$ & 2.26 & 1,7 & 0.177 & $-0.066 \pm 0.064$ & $-0.050 \pm 0.052$ & 0.04 & 1,6 & 0.850 \\
\hline \multicolumn{10}{|l|}{ Body size } \\
\hline Female tarsus length & $\begin{array}{l}a=0.581 \pm 0.068 \\
b=-0.097 \pm 0.018\end{array}$ & 106.40 & 2,8 & $<0.001$ & & & & & \\
\hline Female body mass & $\begin{array}{r}a=0.389 \pm 0.077 \\
b=-0.047 \pm 0.020\end{array}$ & 69.64 & 2,8 & $<0.001$ & & & & & \\
\hline
\end{tabular}

Boldface numbers indicate significant estimates. Difference refers to the interaction between replicate and selection differential. For body size, we estimated nonlinear co-heritabilities, and so $a$ and $b$ represent estimates from $Y=a X+b X^{2}$. 
Table 3 Differences in dry egg components between selection lines

\begin{tabular}{|c|c|c|c|c|c|c|c|c|c|c|c|c|c|}
\hline Trait & Model & $F$ & $d f$ & $P$ & $F$ & $d f$ & $P$ & $F$ & $d f$ & $\mathrm{P}$ & $F$ & $d f$ & $P$ \\
\hline \multicolumn{14}{|c|}{ Dry albumen } \\
\hline & a & 48.20 & 1,77 & $<0.001$ & 0.58 & 1,77 & 0.450 & - & - & - & - & - & - \\
\hline & b & 27.97 & 1,76 & $<0.001$ & 1.24 & 1,76 & 0.268 & 5.28 & 1,76 & 0.024 & - & - & - \\
\hline & c & 4.84 & 1,76 & 0.031 & 0.20 & 1,76 & 0.655 & - & - & - & 125.81 & 1,76 & $<0.001$ \\
\hline \multicolumn{14}{|c|}{ Dry yolk } \\
\hline & a & 17.15 & 1,77 & $<0.001$ & 4.53 & 1,77 & 0.036 & - & - & - & - & - & - \\
\hline & $b$ & 7.82 & 1,76 & 0.007 & 5.12 & 1,76 & 0.026 & 2.57 & 1,76 & 0.113 & - & - & - \\
\hline & c & 8.08 & 1,76 & 0.006 & 3.02 & 1,76 & 0.086 & - & - & - & 223.04 & 1,76 & $<0.001$ \\
\hline \multicolumn{14}{|c|}{ Dry shell } \\
\hline \multicolumn{14}{|c|}{ Total dry mass } \\
\hline & a & 37.95 & 1,77 & $<0.001$ & 4.12 & 1,77 & 0.046 & - & - & - & - & - & - \\
\hline & $b$ & 19.33 & 1,76 & $<0.001$ & 6.10 & 1,76 & 0.016 & 6.74 & 1,76 & 0.011 & - & - & - \\
\hline & c & 0.83 & 1,76 & 0.364 & 7.42 & 1,76 & 0.008 & - & - & - & 917.13 & 1,76 & $<0.001$ \\
\hline
\end{tabular}

For each egg component we ran three analyses, one without covariates (a), one controlling for body size (b) and one controlling for absolute egg size (c). Data from generation 4 are shown. Significant effects are displayed in bold.

increased maternal investment. These could include trade-offs between reproductive investment and immunity (Knowles et al., 2009), predator escape performance (Cooper et al., 1990; Lee et al., 1996) and/or physiological damage, such as oxidative stress (Monaghan et al., 2009). All of these trade-offs would ultimately act to reduce an individual's lifespan. This has been investigated mostly in the context of total reproductive investment, rather than per offspring investment. Also, results have been somewhat inconclusive, with no overall effect of increased reproductive investment on lifespan being found across studies in birds (Santos and Nakagawa, 2012).

Although we found little evidence for genetic constraints limiting the evolution of maternal investment, there was evidence that the response to selection between the two lines was asymmetrical. Between generations 2 and 5, egg size did not increase in the maternal high investment lines but rapidly decreased in the maternal low investment lines. Interestingly, such asymmetrical responses to selection are regularly observed in fitness-related traits (Frankham, 1990).

There are several possible explanations for this phenomenon. Firstly, as we were selecting for egg size relative to body size, it is possible that we reached a physiological and/or morphological maximum. However, this seems unlikely, as females occasionally lay double-yolked eggs of over $21 \mathrm{~g}$ in our population (JLP, personal observation). Secondly, drift, inbreeding depression or different selection pressures can cause asymmetric responses to selection (Frankham, 1990; Falconer and Mackay, 1996). However, we can exclude them all given the design of our experiment. Thirdly, when traits are themselves affected by maternal effects, this can alter their response to selection (Kirkpatrick and Lande, 1989). However these models do not predict the asymmetric responses found in our study. Finally, genetic asymmetry can cause asymmetric responses to selection in fitness-related traits (Frankham, 1990). Genetic asymmetry refers to both directional dominance and unequal allele frequencies (Falconer and Mackay, 1996). The presence of deleterious recessive alleles makes it easy to select downwards, by increasing the frequency of homozygotes for these recessive alleles, but not upwards as the dominant alleles are already expressed in most cases (assuming equal allele frequencies in the base population). In the case of unequal allele frequencies, if the alleles for high fitness are at higher frequency, it similarly becomes difficult to select for higher fitness. Such genetic asymmetries affect the susceptibility of a trait to inbreeding depression, which has, in fact, been observed for egg size in Japanese quail (Sittmann et al., 1966; see Shoffner, 1948; Wang and Pirchner, 1992 for chickens).

In conclusion, we found that egg size responds rapidly to selection along with a correlated response in maternal resource investment. Given the positive effects of maternal resource investment on offspring phenotype (for example, on juvenile size; Krist, 2011), the ability of egg size to evolve will therefore act to accelerate the response to selection of these offspring traits (Kirkpatrick and Lande, 1989; Wolf et al., 1998). We found no evidence for a genetic trade-off between egg size and our indirect measure of fecundity. Furthermore, despite a genetic correlation between egg size and body size, we show that it is possible to successfully select for relative egg size. It is therefore unlikely that selection on body size would constrain the evolution of egg size. Instead, genetic asymmetries may constrain the evolution of larger eggs. Testing for the presence of inbreeding depression in egg size in wild populations, which would provide evidence for such asymmetries, would therefore prove a fruitful next step to understand the mechanisms that shape the evolutionary dynamics of such prenatal maternal effectors.

\section{DATA ARCHIVING}

Data are available from Dryad Digital Repository: http://dx.doi.org/ 10.5061/dryad.gj5b7.

\section{CONFLICT OF INTEREST}

The authors declare no conflict of interest. 


\section{ACKNOWLEDGEMENTS}

We thank Alison Pick and Barbara Schnüriger for help with animal husbandry, Christina Ebneter, Ann-Kathrin Ziegler, Elif Hanic and Mathieu Giraudeau for their help with data collection and Erik Postma and three anonymous reviewers for comments on the manuscript. The study was financially supported by the Swiss National Science Foundation (PP00P3_128386 and PP00P3_157455 to BT).

Bernardo J (1996). The particular maternal effect of propagule size, especially egg size: patterns, models, quality of evidence and interpretations. Am Zool 236: 216-236.

Blanckenhorn WU (2000). The evolution of body size: what keeps organisms small? Q Rev Biol 75: 385-407.

Carey C, Rahn H, Parisi P (1980). Calories, water, lipid and yolk in avian eggs. Condor 82 : 335-343.

Christians JK (2002). Avian egg size: variation within species and inflexibility within individuals. Biol Rev Camb Philos Soc 77: 1-26.

Christians JK, Williams TD (2001). Interindividual variation in yolk mass and the rate of growth of ovarian follicles in the zebra finch (Taeniopygia guttata). J Comp Physiol B 171: 255-261.

Christians JK, Williams TD (2002). Effects of porcine follicle-stimulating hormone on the reproductive performance of female zebra finches (Taeniopygia guttata). Gen Comp Endocrinol 125: 121-131.

Cole LJ (1917). Determinate and indeterminate laying cycles in birds. Anat Rec (Hoboken) 11: 504-505.

Conner JK (2003). Artificial selection: a powerful tool for ecologists. Ecology 84: 1650-1660.

Cooper WE, Vitt LJ, Hedges R, Huey RB (1990). Locomotor impairment and defense in gravid lizards (Eumeces laticeps): behavioral shift in activity may offset costs of reproduction in an active forager. Behav Ecol Sociobiol 27: 153-157.

Czesak ME, Fox CW (2003). Evolutionary ecology of egg size and number in a seed beetle: Genetic trade-off differs between environments. Evolution 57: 1121-1132.

Falconer D, Mackay TF (1996). Introduction to Quantitative Genetics. 4th edn New York: Longman.

Festing M, Nordskog A (1967). Response to selection for body weight and egg weight in chickens. Genetics 55: 219-231.

Finkler MS, Van Orman JB, Sotherland PR (1998). Experimental manipulation of egg quality in chickens: Influence of albumen and yolk on the size and body composition of near-term embryos in a precocial bird. J Comp Physiol B 168: 17-24.

Fischer K, Bot ANM, Brakefield PM, Zwaan BJ (2006). Do mothers producing large offspring have to sacrifice fecundity? J Evol Biol 19: 380-391.

Fox CW, Czesak ME (2000). Evolutionary ecology of progeny size in arthropods. Annu Rev Entomol 45: 341-369.

Frankham R (1990). Are responses to artificial selection for reproductive fitness characters consistently asymmetrical? Genet Res 56: 35.

Freeman BM, Vince MA (1974). Development of the Avian Embryo. London: Chapman and Hall.

Garant D, Hadfield JD, Kruuk LEB, Sheldon BC (2008). Stability of genetic variance and covariance for reproductive characters in the face of climate change in a wild bird population. Mol Ecol 17: 179-188.

Hõrak P, Mänd R, Ots I (1997). Identifying targets of selection: a multivariate analysis of reproductive traits in the great tit. Oikos 78: 592-600.

Henry C, Ulijaszek S (1996). Long-term Consequences of Early Environment. Cambridge: Cambridge University Press.

Hill WG (1972). Estimation of realised heritabilities from selection experiments. I. Divergent selection. Biometrics 28: 747-765.

Houle D (1992). Comparing evolvability and variability of quantitative traits. Genetics 130 195-204.

Hutt F (1949). Genetics of the Fowl. New York: McGraw-Hill.

Johnsgard PA (1988). The Quails, Partridges, and Francolins of the World. Oxford: Oxford University Press.
Kingsolver JG, Hoekstra HE, Hoekstra JM, Berrigan D, Vignieri SN, Hill CE et al. (2001). The strength of phenotypic selection in natural populations. Am Nat 157 245-261.

Kirkpatrick M, Lande R (1989). The evolution of maternal characters. Evolution 43: 485-503.

Knowles SCL, Nakagawa S, Sheldon BC (2009). Elevated reproductive effort increases blood parasitaemia and decreases immune function in birds: a meta-regression approach. Funct Ecol 23: 405-415.

Krist M (2011). Egg size and offspring quality: a meta-analysis in birds. Biol Rev Camb Philos Soc 86: 692-716.

Lee SJ, Witter MS, Cuthill IC, Goldsmith AR (1996). Reduction in escape performance as a cost of reproduction in gravid starlings, Sturnus vulgaris. Proc $R$ Soc Lond $B \mathbf{2 6 3}$ 619-623.

Lessells CM, Cooke F, Rockwell RF (1989). Is there a trade-off between egg weight and clutch size in wild Lesser Snow Geese (Anser c. caerulescens). J Evol Biol 2: 457-472.

Lindström J (1999). Early development and fitness in birds and mammals. Trends Ecol Evol 14: 343-348.

McGinley M, Temme D, Geber M (1987). Parental investment in offspring in variable environments: theoretical and empirical considerations. Am Nat 130: 370-398.

Meijer T (1992). Egg-laying patterns in captive starlings. Ardea 80: 301-310.

Merilä J, Sheldon BC, Kruuk LEB (2001). Explaining statis: microevolutionary studies in natural populations. Genetica 112-113: 199-222.

Miyoshi S, Luc KM, Kuchida K, Mitsomoto T (1996). Selection for high and low yolkalbumen ratio in chickens. V. Effects of selection and relaxation on egg component traits. Jpn Poult Sci 33: 329-338.

Monaghan P, Metcalfe NB, Torres R (2009). Oxidative stress as a mediator of life history trade-offs: mechanisms, measurements and interpretation. Ecol Lett 12: 75-92.

Mousseau T, Fox CW (1998). Maternal Effects as Adaptations. Oxford University Press: Oxford, UK.

Nager RG, Monaghan P, Houston DC (2000). Within-clutch trade-offs between the number and quality of eggs: experimental manipulations in gulls. Ecology 81: 1339-1350.

Nestor KE, Bacon WL, Lambio AL (1983). Divergent selection for egg production in Coturnix coturnix japonica. Poult Sci 62: 1548-1552.

R Core Team (2014). R: A Language and Environment for Statistical Computing. Vienna: R Foundation for Statistical Computing.

Romanoff A, Romanoff A (1949). The Avian Egg. New York: Wiley.

Santos ESA, Nakagawa S (2012). The costs of parental care: a meta-analysis of the tradeoff between parental effort and survival in birds. J Evol Biol 25: 1-7.

Santure AW, De Cauwer I, Robinson MR, Poissant J, Sheldon BC, Slate J (2013). Genomic dissection of variation in clutch size and egg mass in a wild great tit (Parus major) population. Mol Ecol 22: 3949-3962.

Schwarzkopf L, Blows M, Caley M (1999). Life-history consequences of divergent selection on egg size in Drosophila melanogaster. Am Nat 154: 333-340.

Shoffner RN (1948). The reaction of the fowl to inbreeding. Poult Sci 27: 448-452.

Sinervo B, Licht P (1991). Hormonal and physiological control of clutch size, egg size, and egg shape in side-blotched lizards (Uta stansburiana): constraints on the evolution of lizard life histories. J Exp Zoo/ 257: 252-264.

Sittmann K, Abplanalp H, Fraser RA (1966). Inbreeding depression in Japanese quail. Genetics 54: 371-379.

Smith CC, Fretwell SD (1974). The optimal balance between size and number of offspring. Am Nat 108: 499.

Walsh B, Blows MW (2009). Abundant genetic variation + strong selection=multivariate genetic constraints: a geometric view of adaptation. Annu Rev Ecol Evol Syst 40: 41-59.

Wang A, Pirchner F (1992). Course of inbreeding and its effects on feed-efficiency and reproductive traits in laying hens. Arch für Geflügelkd 56: 112-117.

Williams TD (2012). Physiological Adaptations for Breeding in Birds. Princeton: Princeton University Press.

Williams TD, Miller M (2003). Individual and resource-dependent variation in ability to lay supranormal clutches in response to egg removal. Auk 120: 481-489.

Wolf J, Brodie E III, Cheverud J, Moore AJ, Wade M (1998). Evolutionary consequences of indirect genetic effects. Trends Ecol Evol 13: 64-69.

Wright D, Rubin C, Schutz K, Kerje S, Kindmark A, Brandström H et al. (2012). Onset of sexual maturity in female chickens is genetically linked to loci associated with fecundity and a sexual ornament. Reprod Domest Anim 47: 31-36.

Supplementary Information accompanies this paper on Heredity website (http://www.nature.com/hdy) 